\title{
High Blood Pressure Effects on the Brain Barriers and Choroid Plexus Secretion
}

\author{
Ibrahim González-Marrero ${ }^{1}$, Leandro Castañeyra-Ruiz ${ }^{2}$, Juan M. González-Toledo ${ }^{1}$, \\ Agustín Castañeyra-Ruiz ${ }^{2}$, Héctor de Paz-Carmona ${ }^{2}$, Lidia Ruiz-Mayor ${ }^{1}$, \\ Agustín Castañeyra-Perdomo ${ }^{1,2^{*}}$, Emilia M. Carmona-Calero ${ }^{1,2}$ \\ ${ }^{1}$ Departamento de Anatomía, Facultad de Medicina, Universidad de La Laguna, Tenerife, Spain; ${ }^{2}$ Departamento de Biotecnología, \\ Instituto de Investigación y Ciencias de Puerto del Rosario, Fuerteventura, Spain. \\ Email: *acastane@ull.es
}

Received December $6^{\text {th }}, 2011$; revised January $11^{\text {th }}, 2012$; accepted February $24^{\text {th }}, 2012$

\begin{abstract}
High blood pressure produces ventricular dilation, variations in circumventricular organs and changes in the cerebrospinal fluid compositions. On the other hand, chronic hypertension in spontaneously hypertensive rats can cause changes in the integrity of the brain barriers: blood-cerebrospinal fluid barrier and blood brain barrier. The permeability of the brain barriers can be studied by using transthyretin and S-100 $\beta$. In the present work we study the integrity of the brain barrier and the choroid plexus function variations in arterial hypertension. Control rats and spontaneously hypertensive rats were used and the choroid plexus were processed by immunohistochemistry with anti-transthyretin and anti-vasopressin. Western blot was also performed in cerebrospinal fluid, serum and choroid plexus using anti-S-100 $\beta$, antitransthyretin. The accumulation of transthyretin immunoreactive was bigger in spontaneously hypertensive rats with respect to the control. Vasopressin was also higher in spontaneously hypertensive rats with respect to the control. Western blot showed that transthyretin tetramer was higher in the spontaneously hypertensive rats than in the control rats. The expression of transthyretin monomer was lower in hypertensive rats than the control in the cerebrospinal fluid, the transthyretin monomer reaction in the blood was stronger in hypertensive than in control rats. Western blot for the S- $100 \beta$ showed an increase in blood and cerebrospinal fluid of hypertensive rats. The high blood pressure produces a disruption of the blood brain barrier and blood to cerebrospinal fluid barrier that allows extravasations from the cerebrospinal fluid to the blood and from the blood to the cerebrospinal fluid.
\end{abstract}

Keywords: Brain Barriers; Choroid Plexus; Arterial Hypertension

\section{Introduction}

High blood pressure produces ventricular dilation, variations in circumventricular organs and changes in the cerebrospinal fluid (CSF) compositions [1-3]. Arterial hypertension also induces alterations in brain tissue $\mathrm{pH}$, cerebral auto regulation and brain barrier permeability and, a paralysis of cerebral auto regulation may occur suggesting a probable cause of brain edema when the arterial blood pressure fluctuates excessively [4]. The permeability of the brain barriers (BBs) was also investigated in spontaneously hypertensive rats (SHR) $[5,6]$ via studies of transport through the $\mathrm{BB}$, electron microscopy was used to visualize the structural changes that may produce a response to the hypertonic mannitol infusion in both the blood brain barrier (BBB) and blood to CSF barrier (BCSFB) and it was found $[5,6]$, that chronic hypertension in SHR can cause changes in the integrity of the brain barriers,

"Corresponding author. which are more pronounced in the blood -CSF barrier than in the BBB. The permeability of the brain barriers can also be studied by using Transthyretin (TTR) and S-100 $\beta$ : TTR is a protein involved in the transport of thyroid hormones in blood and CSF, and transthyretin is expressed in CSF as a monomer of $14 \mathrm{kDa}$ (TTRm) and a dimmer of $28 \mathrm{kDa}$ (TTRd), and in blood as a tetramer of $55 \mathrm{kDa}$ (TTRt). The TTR is synthesized in the choroid plexus (CP) cells and subcommissural organ (SCO) cells and secreted into the CSF [7-9] and the S- $100 \beta$ is synthesized in the astroglial and Swans cells, and when there are alterations in the $\mathrm{BBB}$, the $\mathrm{S}-100 \beta$ passes into the blood vessels $[9,10]$.

It has been reported that humoral and endotheliumdependent mechanisms may play an important role in cerebral circulation; the brain may sense reductions in microvascular pressure and initiate compensatory neurohumoral responses to raise arterial pressure, and vasopressin II (AV) appears to play an important role in the regula- 
tion of the production of CSF and brain fluid volume $[8,11]$

The aim of the present work is to analyze the integrity of the brain barrier and the choroid plexus function variations in arterial hypertension bearing in mind the connection among arterial hypertension, choroid plexus alterations and brain barrier dysfunction.

\section{Methods}

30 male rats were used, divided into 15 control WistarKyoto rats (WKY) and 15 spontaneously hypertensive rats (SHR) from Charles River Laboratories Spain SA (Barcelona, Spain). The rats were maintained at a constant temperature of $21^{\circ} \mathrm{C} \pm 2{ }^{\circ} \mathrm{C}$ and $55 \% \pm 8 \%$ relative humidity on a normal 12 hour - 12 hour light-dark cycle. The rats were sacrificed at 6 months of age. Ten rats of each group were anesthetized with chloral hydrate (200 $\mu \mathrm{l} / 100 \mathrm{~g}$ of body weight at $160 \mathrm{mg} / \mathrm{ml}$ ) and $100 \mu \mathrm{l}$ of CSF was extracted from the cistern magna and $4 \mathrm{ml}$ of blood was extracted by intracardiac puncture from each animal before sacrifice. The choroid plexus extracts were prepared after sacrifice.

Five rats from each group were fixed by intracardiac perfusion with Bruin's fluid, dehydrated and embedded in paraffin under standard conditions. Brains were cut into four serial coronal sections of $10 \mu \mathrm{m}$ thickness. One of the serial coronal sections was stained with hematoxylineosin (H-E). In the others series, three rostrocaudal-caudal levels of the brain areas containing the $\mathrm{CP}$ of the lateral ventricle were processed immunohistochemicaly as follows: after deparaffinization and rehydration, tissue sections were treated with hot $\left(85^{\circ} \mathrm{C}\right) 10 \mathrm{mM}$ citrate buffer, $\mathrm{pH}$ 6, for $20 \mathrm{~min}$. Sections were washed with distilled water and quenched with $3 \%$ hydrogen peroxide for $10 \mathrm{~min}$ at room temperature to eliminate endogenous peroxidase activity. After washing in $0.05 \mathrm{M}$ Tris-buffered saline $\mathrm{pH}$ 7.6, sections were incubated overnight at $4^{\circ} \mathrm{C}$ with their appropriate primary antibody: rabbit monoclonal anti-transthyretin (TTR) (prealbumin) 1:600 (Dako, Glostrup, Denmark) and anti-vasopressin (AV): 1:2000 (ICN Biomedicals, Inc). After washing the sections were incubated with the biotinylated secondary antibody using the Vectastain Elite ABC Goat Peroxidase system (Vector Laboratories, Burlingame, CA, USA) for $30 \mathrm{~min}$ at room temperature. All tissue sections were washed in TBST and stained using 3.3-diaminobenzidine (DAKO) as the chromogen. Sections were dehydrated through a series of graded alcohols and xylene, and mounted in Eukitt. Primary antibody omission controls were run alongside the other samples to check for non-specific binding due to the secondary antibody, along with positive control tissue.

The CSF, serum and extracts of choroid plexus (ChP) of 10 rats from each group were processed by protein electrophoresis according to Laemmli [12], the proteins were then transferred from gel to nitrocellulose membrane, the membranes were incubated in tris-saline (TBS) non-fat milk $5 \%$ for 60 minutes and were then incubated in the target antibody at a dilution of 1:1000 for Transthyretin (DAKO) and 1:2000 for S-100 $\beta$ (ABCAM) in blocking buffer at $4^{\circ} \mathrm{C}$ overnight. Detection was performed by enhanced chemiluminescence (Amersham Biosciences) after 1 hour incubation with horseradish peroxidase-conjugated anti-rabbit (Jackson). The primary antibody was omitted to validate the control method specificity.

The immunohistochemistry slides were converted to digital images by using a photomicroscope LEICA DMRB with a camera LEICA DC $300 \mathrm{~F}$ (Gemany). Image analysis was completed in Image J (v. $1.43 \mathrm{u}, \mathrm{NIH}$, Bethesda, MD, USA). For all stained tissue and membranes was selected the "Mean Gray Value", that was measured from the selection. This value renders the average stain intensity as grayscale units for all thresholded pixels. An analysis of variance (ANOVA) of single-factor was used for the immunochemistry statistics study, which was conducted using the IBM SPSS statistic 19 software.

\section{Results}

When H-E was used, the normal aspect of the choroids plexus structures in the control WKY appeared as cubic cells abutting each other and with slight structural variations in SHR (Figure 1).

The accumulation of anti-transthyretin inmunoractive (TTR-ir) was mainly found in the cytoplasm of choroid plexus cells and the amount of TTR-ir was significantly bigger $\left(\mathrm{F}_{1-10}=45.917, \mathrm{p}<0.01\right)$ in SHR with respect to the control (Figures 2(a) and (c)). The anti-vasopressin reactions (AV-ir) were also mostly observed in the cytoplasm of the choroid plexus and their concentration was higher $\left(\mathrm{F}_{1-10}=12.160, \mathrm{p}<0.01\right)$ in SHR rats with respect to the control (Figures 2(b) and (c)).

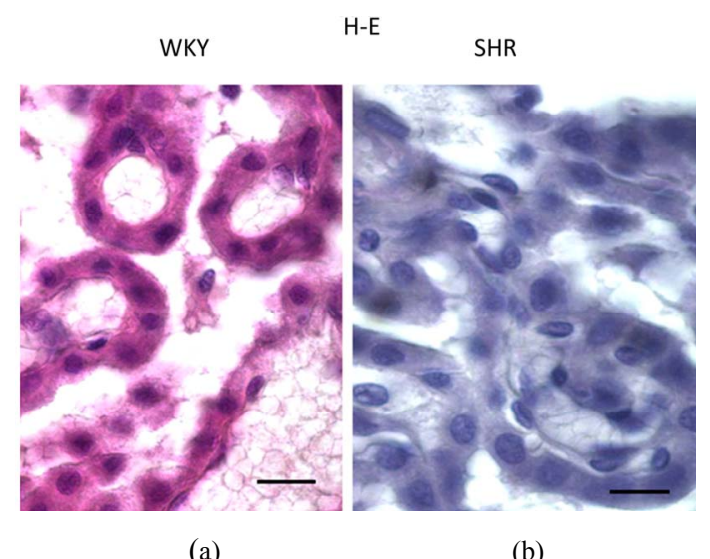

Figure 1. It shows transversal section photographs of the lateral ventricle choroids plexus stained with hematoxylineosin. (a): WKY rats; (b): SHR rats. Bar $=20 \mu \mathrm{m}$. 


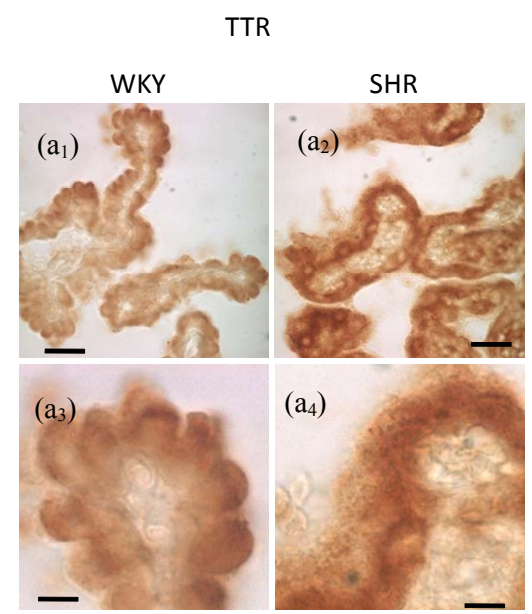

(a)

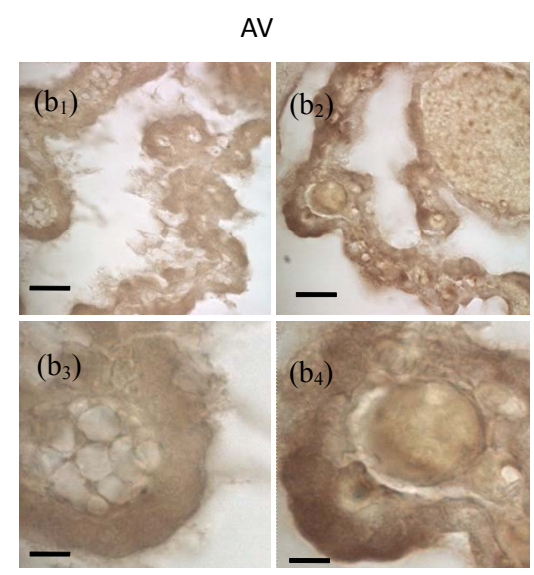

(b)

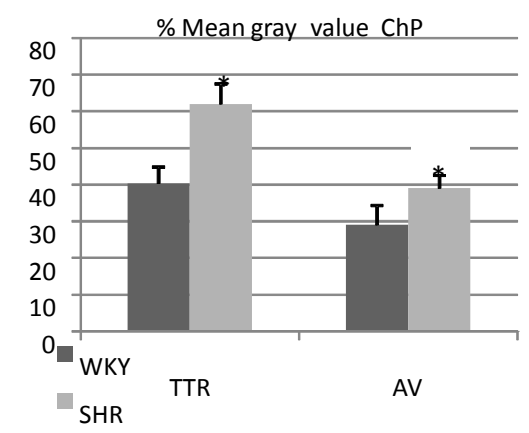

(c)

Figure 2. (a) shows transversal section photographs of the lateral ventricle choroid plexus immunostained with antitransthyretin (TTR). $\left(a_{1}\right)$ and $\left(a_{3}\right)$ WKY rats. $\left(a_{2}\right)$ and $\left(a_{4}\right)$ SHR rats. Bar: $\left(a_{1}\right),\left(a_{2}\right)=25 \mu \mathrm{m},\left(a_{3}\right),\left(a_{4}\right)=10 \mu \mathrm{m} ;(b)$ shows transversal section photographs of the lateral ventricle choroid plexus immunostained with anti-vasopressin (AV). $\left(b_{1}\right)$ and $\left(b_{3}\right)$ WKY rats. $\left(b_{2}\right)$ and $\left(b_{4}\right)$ SHR rats. Bar: $\left(b_{1}\right)$, $\left(b_{3}\right)=25 \mu \mathrm{m},\left(b_{2}\right),\left(b_{4}\right)=10 \mu \mathrm{m}$; (c) shows the "Mean Gray Value" statistical analysis and graphs of the slides containing the choroid plexus immunohistochemically processed for TTR and AV. *TTR: $F_{1-10}=45.917, p<0.01 ; * A V: F_{1-10}$ $=12.160, \mathrm{p}<0.01 ;$ TTR $=$ transthyretin, $\mathrm{AV}=$ vasopressin WKY $=$ control WistarKyoto rats SHR $=$ spontaneously hypertensive rats.
The western blot of the choroid plexus extract showed that TTR dimmer (TTRd) did not vary in the control and hypertensive rats (Figure 3(a)), but TTR monomer (TTRm) was higher $\left(\mathrm{F}_{1-10}=14.98, \mathrm{p}<0.01\right)$ in the SHR than in the WKY rats (Figure 3(a)). The TTR tetramer (TTRt) was also higher in the SHR than in the WKY rats (Figure 3(a)).

The western blot of CSF and blood showed that, the expression of TTRm was lower $\left(\mathrm{F}_{1-10}=299.5, \mathrm{p}<0.01\right)$ in hypertensive rats than the control in the CSF (Figure 3(b)), the TTRm reaction in the blood was stronger $\left(\mathrm{F}_{1-10}\right.$ $=41, \mathrm{p}<0.01)$ in hypertensive rats than in control WKY rats (Figure 3(b)). TTR dimmer (TTRd) did not vary in the control and hypertensive rats (Figure 3(c)).

Western blot for the $\mathrm{S}-100 \beta$ showed a marked increase in blood $\left(\mathrm{F}_{1-10}=75.72, \mathrm{p}<0.01\right)$ and $\operatorname{CSF}\left(\mathrm{F}_{1-10}=37.01\right.$, $\mathrm{p}<0.01)$ of hypertensive rats when compared with controls rats (Figure 4).

\section{Discussion}

In previous works, we described alterations in several circumventricular organs, ventricular dilation and variations in the protein composition of the CSF in spontaneously hypertensive rats [1-3].

On the other hand, Capone et al. [13] have reported that the alterations in neurovascular regulation induced by slow pressor angiotensin II develop before hypertension and persist beyond arterial pressure normalization but are not permanent. The findings unveil a striking susceptibility of cerebrovascular function to the deleterious effects of ANG II and raise the possibility that cerebrovascular dysregulation precedes the elevation in arterial presusre in patients with angiotensin II (ANG II)-dependent hypertension [13]. Furthermore, other works indicate that ANG II and the vasopressin together with humoral and endothelium-dependent mechanisms may play an important role in the cerebral circulation and in the regulation of the production of cerebrospinal fluid and brain fluid volume $[8,11]$. As regards vasopressin (AV), the results here show that $\mathrm{AV}$ decreased in hypertensive rats which could signify a decrease in the vascular regulation in the CP of these rats.

The TTR monomer (m) is synthesized in the CP cells and secreted into the CSF. Some authors have described that TTRm is also synthesized in the SCO cells and is secreted from here into the CSF [7-9]. In the results of the present work it was found that when using immunohistochemistry and western blot there is an increase of TTR in hypertensive rats with respect to the control in the CP, this finding could mean alterations in the TTR secretions by CP cells.

The choroid plexus (CP) of the blood-CSF barrier (BCSFB) shows basically different properties to bloodbrain barrier. The human $\mathrm{CP}$ provides the $\mathrm{CNS}$ with a high 


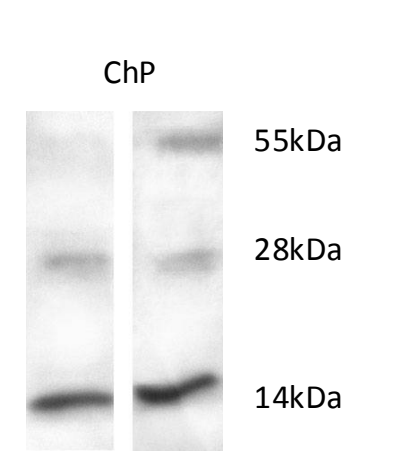

\section{TTR}

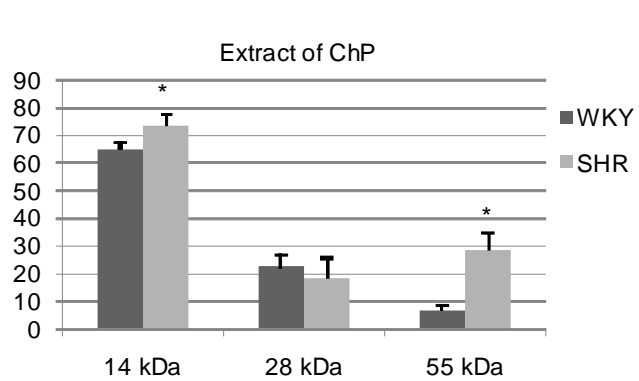

(a)
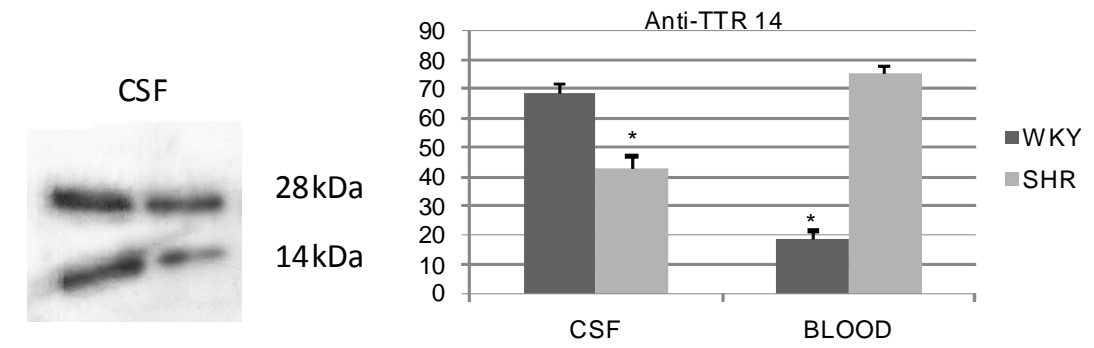

(b)
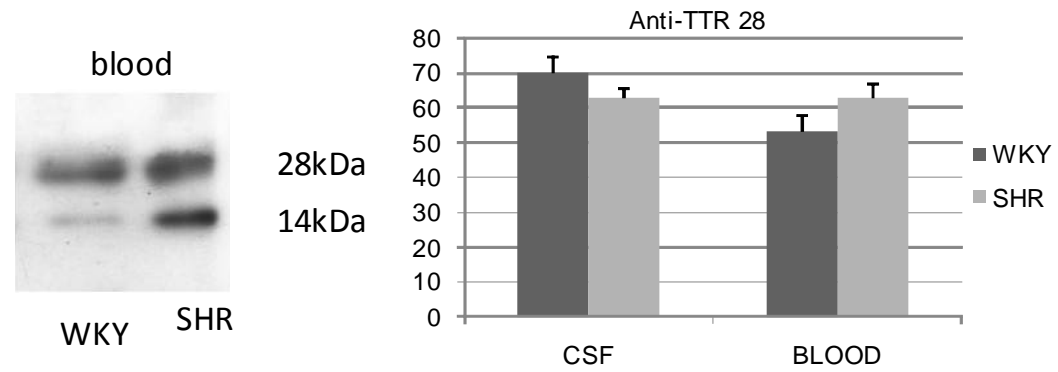

(c)

Figure 3. It shows the western blot, the "Mean Gray Value" statistical analysis and graphs of the western blot study for TTR: (a) in extracts of choroid plexus, ${ }^{*} F_{1-10}=14.98, p<0.01$ in TTRm, ${ }^{*} F_{1-10}=53.46, p<0.01$ in TTRd; (b) in the CSF; (c) in the blood. TTR = transthyretin, TTRm = TTR monomer $(14 \mathrm{kDa})$, TTRd $=$ TTR dimmer $(28 \mathrm{kDa})$, TTRt $=$ TTR tetramer $(55$ $\mathrm{kDa}), \mathrm{WKY}=$ control Wistar-Kyoto rats, $\mathrm{SHR}=$ spontaneously hypertensive rats, $\mathrm{kDa}=$ Kilo-Dalton, $\mathrm{CSF}=$ cerebrospinal fluid.
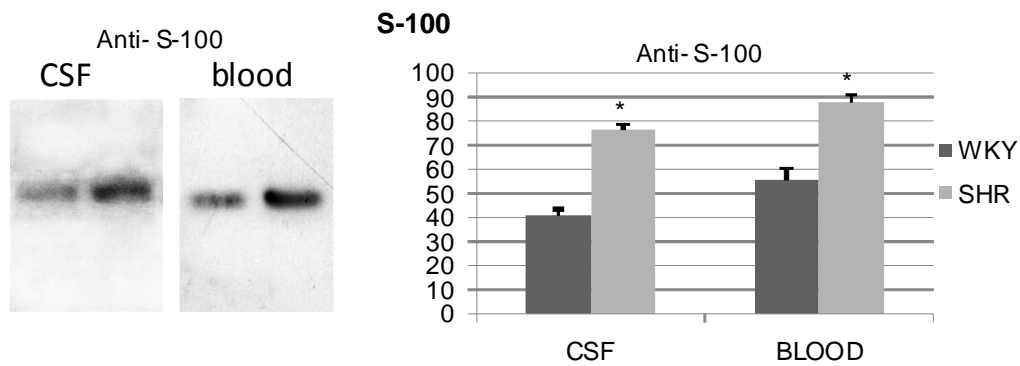

Figure 4. It shows the western blot, the "Mean Gray Value" statistical analysis and graph of the western blot study for S-100 $\beta$ in the CSF and blood (serum). ${ }^{*} F_{1-10}=75.72, p<0.01$ in CSF, ${ }^{*} F_{1-10}=37.01, p<0.01$ in blood.

turnover rate of fluid containing peptides and hormones the brain with rapid blood flow and highly permeable capillaries [14]. Al-Sarraf and Philip [5] have reported an increased permeability of the blood-CSF barrier with sucrose in chronic hypertensive rats, the results here agree with this finding since it was seen that the TTRm, which is usually present in CSF but scarce or undetectable in blood, is increased in blood and decreased in the CSF in SHR rats when compared with WKY. But with respect to the $\mathrm{BBB}$, they [5] described that the $\mathrm{BBB}$ was resistant to the passage of sucrose and lanthanum in both SHR and WKY rats, indicating that the BBB integrity was main- 
tained although they report, at the end, that chronic hypertension in SHR may cause more pronounced defects in the integrity of the BCSFB than in the BBB [5]. We have observed that the $\mathrm{S}-100 \beta$, which is a protein used for studying BBB permeability $[9,10]$, was clearly increased in blood meaning a clear disruption of the BBB in SHR rats.

Therefore, we conclude that the arterial hypertension in these rats cause cellular function alterations in the $\mathrm{CP}$ structure which induce variations in protein secretion into the CSF, and high blood pressure produces a disruption of the BBB and BCSFB, expressed by an increase of S- $100 \beta$ and TTRm in the blood that allows extravasations from the CSF to the blood and from the blood to the CSF.

\section{Acknowledgements}

This work was supported by the Fundación Canaria de Instituto de Investigacion y Ciencias de Puerto del Rosario (INIPRO) project No. 01/10 and Project No. 02/10.

\section{REFERENCES}

[1] I. Martínez-Peña y Valenzuela, E. M. Carmona-Calero, H. Pérez-González, C. Ormazabal-Ramos, P. Fernández- Rodríguez, I. González-Marrero, A. Castañeyra-Perdomo and R. Ferres-Torres, "Alterations of the Cerebrospinal Fluid Proteins and Subcommissural Organ Secretion in the Arterial Hypertension and Ventricular Dilatation. A Study in SHR Rats," Histology and Histopathology, Vol. 21, No. 2, 2006, pp. 179-185.

[2] I. González-Marrero, E. M. Carmona-Calero, P. Fernández-Rodríguez, H. Pérez-González, C. OrmazabalRamos, L. Castañeyra-Ruiz, C. G. Pérez-García, I Martínez-Peña-Valenzuela, A. Castañeyra-Ruiz, A. CastañeyraPerdomo and R. Ferres-Torres, "Expression of Certain Proteins in the Subfornical Organ and Cerebrospinal Fluid of Spontaneously Hypertensive Rats," Histology and Histopathology, Vol. 22, No. 12, 2007, pp. 1371-1378.

[3] E. M. Carmona-Calero, H. Perez-Gonzalez, I. MartinezPeña y Valenzuela, I. Gonzalez-Marrero, C. G. PerezGarcia, N. Marrero-Gordillo, C. Ormazabal-Ramos, A. Castañeyra-Perdomo and R. Ferres-Torres, "Effect of the Arterial Hypertension and Captopril Treatment on the Angiotensin II Content in the Subfornical Organ. A Study in SHR Rats," Histology and Histopathology, Vol. 20, No. 1, 2005, pp. 135-138.

[4] H. Katsumura, M. Kabuto, K. Hosotani, Y. Handa, H. Kobayashi and T. Kubota, "The Influence of Total Body Hyperthermia on Brain Haemodynamics and Blood-Brain
Barrier in Dogs," Acta Neurochir (Wien), Vol. 135, No. 1-2, 1995, pp. 62-69. doi:10.1007/BF02307416

[5] H. Al-Sarraf and L. Philip, "Effect of Hypertension on the Integrity of Blood Brain and Blood CSF Barriers, Cerebral Blood Flow and CSF Secretion in the Rat," Brain Research, Vol. 975, No. 1-2, 2003, pp. 179-188. doi:10.1016/S0006-8993(03)02632-5

[6] H. Al-Sarraf, F. Ghaaedi and Z. Redzic, "Time Course of Hyperosmolar Opening of the Blood-Brain and BloodCSF Barriers in Spontaneously Hypertensive Rats," Journal of Vascular Research, Vol. 44, No. 2, 2007, pp. 99109. doi: $10.1159 / 000098260$

[7] H. A. Montecinos, H. Richter, T. Caprile and E. M. Rodríguez, "Synthesis of Transthyretin by the Ependymal Cells of the Subcommissural Organ," Cell and Tissue Research, Vol. 320, No. 3, 2005, pp. 487-499. doi:10.1007/s00441-004-0997-0

[8] A. Chodobski, G. D. Silverberg and J. SzmydyngerChodobska, "The Role of the Choroid. Plexus in the Transport and Production of Polypeptides," In: W. Zheng and A. Chodobski, Eds., The Blood-Cerebrospinal Fluid Barrier, Chapman \& Hall/CRC Taylor \& Francis Group, Boca Raton, 2005, pp. 242-278.

[9] N. Marchi, V. Fazio, L. Cucullo, K. Kight, T. Masaryk, G. Barnett, M. Vogelbaum, M. Kinter, P. Rasmussen, M. R. Mayberg and D. Janigro, "Serum Transthyretin Monomer as a Possible Marker of Blood-to-CSF Barrier Disruption," Journal of Neuroscience, Vol. 23, No. 5, 2003, pp. 1949-1955.

[10] B. J. Blyth, A. Farhavar and C. Gee, "Validation of Serum Markers for Blood-Brain Barrier Disruption in Traumatic Brain Injury," Journal of Neurotrauma, Vol. 26, No. 9, 2009, pp.1497-1507. doi:10.1089/neu.2008.0738

[11] F. M. Faraci and D. D. Heistad, "Regulation of Cerebral Blood Vessels by Humoral and Endothelium-Dependent mechanisms. Update on Humoral Regulation of Vascular Tone," Hypertension, Vol. 17, No. 6, 1991, pp. 917-922.

[12] U. K. Laemmli, "Cleavage of Structural Proteins during the Assembly of the Head of Bacteriophage T4," Nature, Vol. 227, No. 5259, 1970, pp. 680-685. doi: $10.1038 / 227680 \mathrm{a} 0$

[13] C. Capone, G. Faraco, L. Park, X. Cao, R. L. Davisson and C. Iadecola, "The Cerebrovascular Dysfunction Induced by Slow Pressor Doses of Angiotensin II Precedes the Development of Hypertension," American Journal of Physiology, Vol. 300, No. 1, 2011, pp. H397-H407.

[14] C. E. Johanson, E. G. Stopa and P. N. McMillan, "The Blood-Cerebrospinal Fluid Barrier: Structure and Functional Significance," Methods in Molecular Biology, Vol. 686, 2011, pp. 101-131. doi:10.1007/978-1-60761-938-3 4 